\title{
Kinematic analysis of handwriting movements in patients with obsessive-compulsive disorder
} P Mavrogiorgou, R Mergl, P Tigges, J El Husseini, A Schröter, G Juckel, M Zaudig,
U Hegerl
Laboratory of Clinical Neurophysiology and Outpatient Clinic for Obsessive-Compulsive Disorder, Department of Psychiatry,

Ludwig-MaximiliansUniversität München, Nussbaumstrasse 7, D-80336 München,

Germany

P Mavrogiorgou

R Mergl

P Tigges

J El Husseini

A Schröter

G Juckel

U Hegerl

Psychosomatic Hospital Windach, Schützenstraße 16, D-86949 Windach, Germany

M Zaudig

Correspondence to: Dr Roland Mergl

Roland.Mergl@

psy.med.uni-muenchen.de

Received 12 May 2000 and in revised form

14 November 2000

Accepted 8 December 2000
Abstract

Objectives-Basal ganglia dysfunction is supposed to play a part in the pathophysiology of obsessive-compulsive disorder (OCD). A new computer aided technique for the analysis of hand movements, allowing the detection of subtle motor performance abnormalities, was applied in this study of patients with OCD and healthy controls.

Methods-Using a digitising graphic tablet, hand motor performance was studied in 22 unmedicated patients with OCD and compared with 22 healthy controls. All subjects drew superimposed concentric circles with both the right and the left hand, in addition to writing a given sentence, their personal signature, and letter sequences in four different sizes. Kinematic parameters were calculated to quantify hand motion.

Results-Patients with OCD had significant impairments of handwriting performance, reflected by lower peak velocity (sentence $t=3.6 ; p=0.001$; signature $t=2.8$; $\mathrm{p}=0.01$ ) and micrographia (sentence $t=3.4 ; \mathrm{p}=0.002 ;$ signature $t=2.5 ; \mathrm{p}=0.02)$, compared with controls and shortened acceleration phases per stroke (sentence $t=2.4 ; \mathrm{p}=0.02 ;$ signature $t=4.1 ; \mathrm{p}=0.000)$. By contrast, in repetitive drawing, patients with OCD had higher peak velocity than healthy controls (group $\times$ task interaction $p<0.01)$. There were no significant differences in left and right hand performance between groups. Patients with early versus late age of onset differed in handwriting parameters, such as handwriting consistency. Greater severity of obsessions and compulsions correlated with increasingly poor handwriting performance in patients with OCD.

Conclusions-A subtle motor dysfunction in OCD can be detected with a digitising tablet. The findings show handwriting impairments in patients with OCD, in line with the assumption that basal ganglia dysfunction is part of OCD pathophysiology. Repetitive motor pattern performance was not impaired, but rather tended to be even better in patients with OCD than in controls. The findings also support the concept that patients with $O C D$ with early versus late age of onset differ in pathophysiological mechanisms and basal ganglia dysfunction.

(F Neurol Neurosurg Psychiatry 2001;70:605-612)
Keywords: obsessive-compulsive disorder; digitising tablet; handwriting

Obsessive-compulsive disorder (OCD) is a common psychiatric disease, with a lifetime prevalence of $2 \%-3 \% .^{1}$ The hallmark symptoms of OCD include intrusive thoughts (obsessions) as well as ritualistic behaviours (compulsions). According to one of the predominant neurobiological hypotheses, OCD is caused by a basal ganglia dysfunction. ${ }^{2}$

Reports suggestive of dysfunction of the basal ganglia in patients with OCD include findings from CT and MRI showing reduced caudate volume in a subgroup of patients with OCD ${ }^{34}$ the association of OCD with basal ganglia disorders such as Sydenham's chorea ${ }^{5}$ or Gilles de la Tourette's syndrome, ${ }^{6}$ and an increased incidence of movement disorders caused by the basal ganglia among patients with OCD. ${ }^{7}$

A large range of motor disturbances is associated with OCD psychopathology, including catatonic signs, ${ }^{8}$ motor slowness ${ }^{9}{ }^{10}$ and hyperkinesia. ${ }^{11}$ Abnormalities of fine motor coordination and involuntary movements-typical neurological "soft" signs-were also found in about $30 \%$ of patients with OCD. ${ }^{12}$ Several studies have shown that a subset of patients with OCD is characterised by an increase in these signs. ${ }^{13} 14$ Furthermore, some findings suggest that the signs may be a biological marker of a subgroup of patients with OCD with higher neuropsychiatric impairment than other patients with this disorder: patients with OCD with more neurological soft signs had more severe obsessions and a decreased response to treatment with serotonin reuptake inhibitors. ${ }^{13}$ In addition, several studies suggest a lateralisation of neurological soft signs to the right hemisphere. ${ }^{49}$

Investigations of motor abnormalities in patients with OCD can contribute to the evaluation of the importance of neurological soft signs in OCD pathophysiology. However, they require a more rigorous methodology than has been used so far. A new computer aided technique for the measurement and analysis of hand movements while writing and drawing enables objective studies of motor performance in healthy subjects ${ }^{15}$ and patients with psychiatric diseases such as schizophrenia, ${ }^{16}$ depression, ${ }^{17}$ and dementia. ${ }^{18}$

In this study, the kinematic analysis of hand movements was used to compare the motor performance of patients with OCD with that of healthy controls. The main aim was to confirm the hypothesis that patients with OCD write 
and draw slower and less automatically than controls. Another aim was to determine if there exists a lateralisation of hand motor dysfunction towards the right cerebral hemisphere in patients with OCD. If this were the case, differences between the dominant and the nondominant hand in motor performance for velocity, consistency of velocity, and the degree of disturbances of automatisation would be expected to be significantly higher in patients with OCD than in controls. The extent to which hand movement dynamics is modulated by clinical variables (age at onset of disease, severity of obsessions, and depression) in patients with OCD was also investigated.

\section{Methods}

SUBJECTS

Twenty two adult patients between the ages of 17 and 57 (mean age 33.6 (SD 11.8) years) (10 men and 12 women), who met DSM-III-R criteria for OCD, participated in the study. Twenty patients were right handed, the other two patients were left handed (determined by the Edinburgh handedness inventory ${ }^{19}$ ). The patients' verbal intelligence was estimated with the Wortschatztest ${ }^{20}$ ((WST) mean IQ score 106.9 (SD 11.9))

All patients gave written consent according to the guidelines found in the Declaration of Helsinki. ${ }^{21}$ Most of the patients were being treated in the Psychosomatic Hospital Windach at the time of the study. Some of them had been referred to an outpatient clinic specialising in the treatment of OCD and in the long term prophylaxis of affective disorders, localised in the department of psychiatry (LudwigMaximilians-Universität (LMU), München). Duration of disease ranged from 1.5 to 30 years (mean duration 10.8 (SD 6.7) years), the patients' mean age at the beginning of disease was 22.2 (SD 10.7) years. The severity of OCD symptoms was assessed using the Yale-Brown obsessive-compulsive scale (Y-BOCS) ${ }^{22}{ }^{23}$ and the severity of concomitant depression using the 17 item version of the Hamilton depression rating scale (HDRS) ${ }^{24}$ and the Beck depression inventory (BDI). ${ }^{25}$ The patients had a mean total Y-BOCS score of 17.6 (SD 7.9), their Y-BOCS score for obsessions being 9.6 (SD 5.8 ) and their Y-BOCS score for compulsions 8.1 (SD 5.1). Their mean HDRS score was 17.4 (SD 13.2) and their mean BDI score was 11.5 (SD 7.3). All patients with OCD were medication free for 14 days previous to and during testing; some of them had never received psychotropic drugs. No patient had taken long acting psychotropic drugs known to effect motor control several weeks after termination of treatment (for example, neuroleptic drugs). The patients with OCD had neither focal neurological disease nor any other major medical disorder at the time of testing.

Normal controls $(n=22$; nine men and 13 women; 21 right handers and one left hander; mean WST score 113.6 (SD 13.8)), with a mean age of 34.9 (SD 13.5) years, were comparable with the patients for sex $\left(\chi^{2}(1\right.$, $\mathrm{n}=44)=0.82 ; \mathrm{p}=0.37)$, handedness $\left(\chi^{2} \quad(1\right.$, $\mathrm{n}=44)=0.36 ; \quad \mathrm{p}=0.55), \quad$ age $\quad(t(42)=0.33$; $\mathrm{p}=0.74$ ), and verbal intelligence (WST IQ) $(t(42)=1.73 ; \mathrm{p}=0.09)$. The last two variables were found to have a marked influence on the hand motor performance of healthy subjects. ${ }^{15}$ The patients with OCD and controls also had comparable occupational profiles, with six patients and 10 controls being pupils or students; five patients and two controls working as secretaries, clerks, etc executing predominantly writing activities; three patients and six controls working in the public health system as physicians, nurses etc; four patients and one control working in commerce (as businessmen, clerks etc); and four patients and three controls predominantly working with coarse motor activities (as workers, housewives etc) (continuity coefficient $=0.35 ; \mathrm{p}=0.27$ ). There were also no significant differences between patients with OCD and healthy controls for the mean duration of handwriting per day as reported by the subjects (patients with OCD 69.8 (SD 65.1) minutes per day; controls 55.4 (SD 58.3) minutes per day; Mann-Whitney $U=190 ; \mathrm{p}=0.32)$.

All healthy controls were volunteers recruited by researchers in the department of psychiatry (LMU München) and tested in the laboratory of clinical neurophysiology at that institution. They were free of medication and any DSM-III-R diagnosis of mental disorders. No control had neurological or medical illness at the time of examination.

To test the hypothesis of a lateralisation of hand motor performance in patients with OCD towards the right cerebral hemisphere, we compared the hand motor performance of all right handed patients we had investigated with that of the right handed healthy controls. The groups did not differ significantly in age, sex, verbal intelligence assessed by WST scores, occupational profiles, or mean duration of handwriting per day (in minutes). The mean OCD intensity scores for the right handed patients on the Y-BOCS was 17.1 (SD 7.9), for obsessions 9.3 (SD 5.8) and for compulsions 7.9 (SD 5.1). The average HDRS score for the patients was 10.9 (SD 7.1). The patients' mean age at the onset of disease was 22.7 years (SD 10.6 years); their mean illness duration was 11.2 years (SD 7.0 years).

\section{APPARATUS AND TASK}

The subjects first answered a questionnaire to determine relevant characteristics for the analysis of hand movements. Then they filled out the Edinburgh handedness inventory. ${ }^{19}$ The general level of verbal intelligence was assessed with WST. ${ }^{20}$

A digitising graphic tablet (WACOM IV), with a sampling rate of maximally $200 \mathrm{~Hz}$ and a spatial resolution of $0.05 \mathrm{~mm}$, was used to record the $x-y$ coordinates of the subjects' hand movements. The tablet was connected to a personal computer. The control of trials, the registration of data, and the subsequent signal processing were done with a commercially available program $\left(\mathrm{CS}^{26}\right)$.

$\mathrm{Jahn}^{27}$ has rigorously tested the above mentioned equipment and software in the analysis of fine motor diadochokinesia in 
healthy subjects (repetitive drawing of superimposed concentric circles as fast as possible) and found that the internal consistencies of the kinematic CS parameters computed separately for different coordinates and directions were high $(r>0.8)$. Two week stabilities of velocity parameters reflecting diadochokinesia, such as frequency, mean peak velocity, and mean peak acceleration, were found to be in the middle range $(0.67<r<0.72)$. In our own study ${ }^{15}$ we found 1 week stability values for these parameters to be within a similar range for healthy volunteers ( $r$ about 0.6 ). Higher test-retest reliability coefficients $(r>0.7)$ were calculated for handwriting movement parameters, especially mean peak velocity and acceleration and their variation coefficients.

Hand movement samples were taken in a quiet room of the department of psychiatry of the LMU, Munich, during the day. It was not possible to carry out the investigation at a fixed time because many of the patients with OCD were outpatients and most of the healthy volunteers were not employed at the department of psychiatry. The subjects were seated in an armchair, with an adjustable back, with the digitising tablet in front of them on the top of a desk. Every participant could adjust the chair into a comfortable position. The instructor sat beside the subject, in front of a PC.

The subjects had to carry out the following tasks:

- Firstly they were asked to draw concentric superimposed circles with a diameter of $12 \mathrm{~mm}$ between two marked parallel and horizontal lines on a plastic foil to reduce friction, laying over a white sheet of paper (size $29.7 \times 21 \mathrm{~cm}$ ). They had to perform this task in a natural writing position as fast as possible with their dominant hand for 30 seconds to register their degree of fine motor dysdiadochokinesia. Three segments (5-10 seconds; 15-20 seconds; $25-30$ seconds) were analysed and compared to investigate the effects of fatigue on hand motor performance in patients and controls (not reported in this article); the corresponding kinematical variables were then averaged for further statistical analysis.

- To assess hand motor asymmetry, the subjects carried out the same task using their nondominant hand after performance of some handwriting tasks described below; the same three segments were analysed as above.

- The subjects were also asked to write the given sentence: "Der Ball rollt ins Tor" ("The ball rolls into the goal") with their dominant hand on a marked horizontal line in about the middle of a white sheet of paper. The subjects were neither asked to write as neatly as possible nor to write as quickly as possible. The instruction was to write as usual in a natural writing position.

- Then the subjects were asked to write their personal signature as usual with their dominant hand on a marked horizontal line on another white sheet of paper; calligraphic aspects or velocity were not specified.

- The third handwriting task was to write the three letters "aaa" with the dominant hand in a row, using cursive handwriting, without raising the ball point pen used for writing. The letter sequences had to be drawn in a relaxed and fluent manner between two parallel horizontal lines on a separate white sheet of paper in four different sizes $(6,12,18$, and $24 \mathrm{~mm})$. The instructor told the subjects that velocity and calligraphy were not relevant in this task.

All subjects wrote and drew with a special ball point pen with magnetic sensors. They received visual feedback on hand movements while writing and drawing. There were no complete practice runs before the test runs, because the tasks were simple. In some cases, the instructor demonstrated the task before recording the subjects' hand movements. The patients with OCD had an opportunity to write and draw superimposed circles on an extra sheet of paper before their corresponding hand movements were recorded. Several patients did so, mostly before the assessment of the degree of their fine motor diadochokinesia. Kinematic variables were only computed for hand movements executed on paper while writing with the dominant hand.

The velocity and acceleration curves for handwriting were computed for the $\mathrm{x}$ and $\mathrm{y}$ coordinates, but only data for the y coordinate are presented. Kinematic data computed separately for the $\mathrm{x}$ and $\mathrm{y}$ coordinates have been shown to be equivalent, ${ }^{27}$ but the data computed for the y coordinate is graphically more illustrative.

In CS, non-parametric kernel estimation was used for the calculation and smoothing of the derivation. ${ }^{28}$

To determine the different quantitative variables of motion, the coordinates of handwriting movement and its first and second derivations (velocity and acceleration) were divided into time segments, called strokes.

A stroke was defined by the distance between two sequential extreme scores (maxima or minima) of the respective coordinates. These scores corresponded to 0 points of velocity. Similarly, the limits of a segment for velocity were also 0 points of acceleration. These points are identical with the extreme scores of velocity.

For subjects and trials, the following motion parameters were determined: number of changes of direction of velocity (NCV) per stroke; arithmetic mean of stroke peak velocity $(\mathrm{mm} / \mathrm{s})$, with the respective variation coefficient (percentage quotient obtained by dividing absolute SD of peak velocity by mean peak velocity - that is, peak velocity inconsistency); mean stroke length (mm) for the given sentence and the personal signature with the respective variation coefficient (stroke length inconsistency), computed only for the handwriting tasks because handwriting size has not been specified; and skewness coefficient (\%), the ratio of the acceleration phase to the total movement time.

Movement parameters permit exact statements on the quality of handwriting processing. The number of changes of movement is a measure of the degree of movement automatisation. Highly automatised hand movements, such as skilled handwriting, are represented by 


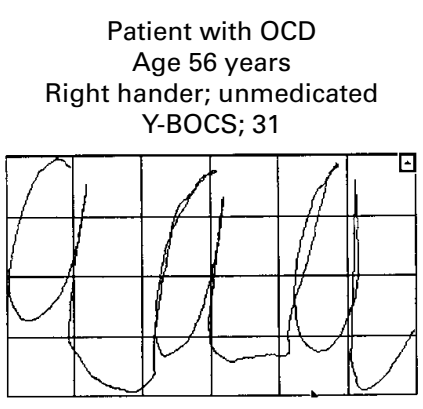

Amplitude
(mm)

Velocity
$(\mathrm{mm} / \mathrm{s})$

Acceleration

Amplitude

( $\mathrm{mm}$ )

Healthy control

Age 55 years

Right hander; unmedicated

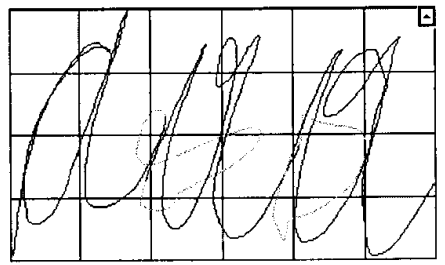

Velocity

(mm/s)

Acceleration $\left(\mathrm{mm} / \mathrm{s}^{2}\right)$ $\left(\mathrm{mm} / \mathrm{s}^{2}\right)$
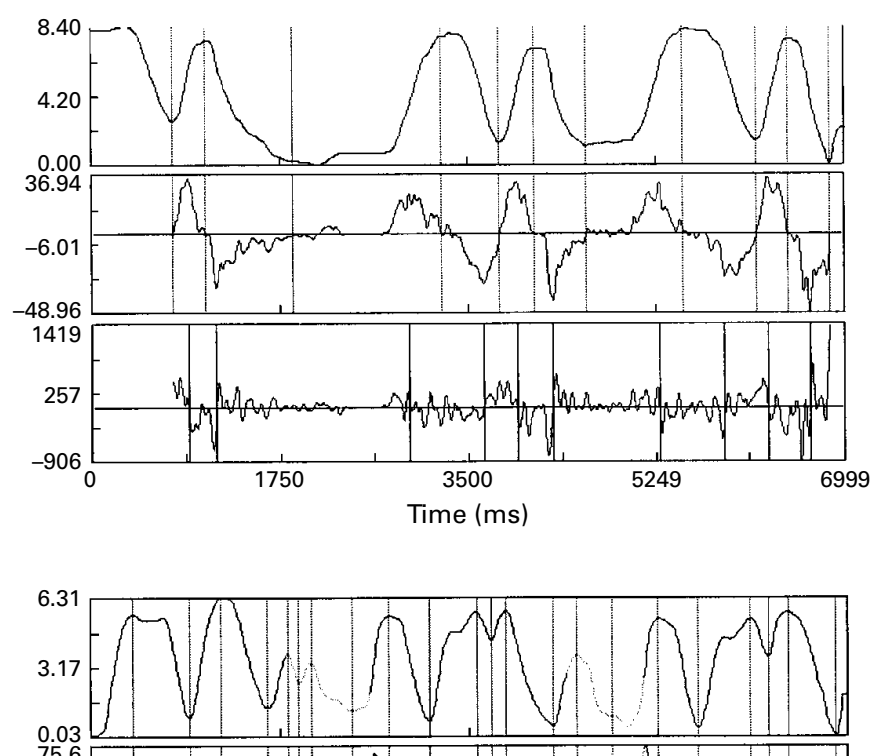

Figure 1 Temporal segmentation of the amplitude and related velocity and acceleration profiles for a patient with OCD and a healthy control. Both subjects wrote three letters ("aaa") between two horizontal lines at a distance of $6 \mathrm{~mm}$. The patient needed more time to perform this task than the control (frequency $0.82 \mathrm{~Hz}$; control $2.81 \mathrm{~Hz}$ ); only the kinematic analyses of his velocity and acceleration profiles show that they are much less regular than those of the control.

bell shaped curves corresponding to monotonous acceleration before and deceleration after the velocity peak of every single stroke within a continuous movement pattern. The number of changes of direction of velocity per stroke is ideally $1 .{ }^{29}$ Figure 1 illustrates the low degree of handwriting automatisation in a patient with OCD, as reflected by a high number of inversions of his velocity profile (for further characteristics of this patient see appendix). By contrast, hand movement automatisation in a healthy control performing the same task (drawing of three letter triples ("aaa") $6 \mathrm{~mm}$ high) is characterised by fewer inversions of velocity, or more bell shaped velocity and acceleration curves.

To determine the regularity of movement processes, the variation coefficients of kinematic parameters can be used. The ideal score is 0 , if the movement replications are identical. Mean peak velocity is suited for the quantification of relative decelerations and accelerations of handwriting movements. Mean peak velocity reflects bradykinesia, the average stroke length micrographia or macrographia, and the skewness coefficient, "the relative proportion of time spent in acceleration during the writing stroke" (Galucci et $a l^{30} \mathrm{p}$ 831). All these parameters were computed by CS.

The results were then statistically analysed with the statistical program SPSS (version 7.5).

\section{Results}

HAND MOTOR PERFORMANCE IN PATIENTS WITH OCD AND CONTROLS

Patients with OCD and controls were compared for differences in the kinematic parameters of circle drawing and handwriting tasks (sentence, signature, letter triples).

Patients with OCD had significantly lower peak velocity while writing a sentence (mean $=57.73 \mathrm{~mm} / \mathrm{s}$ ) and their signature (mean $=77.82 \mathrm{~mm} / \mathrm{s}$ ) than healthy subjects (mean $=81.52 \mathrm{~mm} / \mathrm{s}$ and $112.5 \mathrm{~mm} / \mathrm{s}$ respectively) (fig 2 A). Also, handwriting strokes (sentence and signature) were shorter, reflecting micrographia in patients with OCD compared with controls (fig $2 \mathrm{~B}$ ). The proportion of time spent in acceleration during the writing of a given sentence and the signing of a personal signature was significantly lower in patients with OCD than in healthy controls (fig $2 \mathrm{C}$ ). The last finding shows shortened acceleration phases in the handwriting strokes of patients with OCD. There were no significant differences between the two groups in any other kinematic parameter or in any other task.

Multivariate variance analyses showed that hand motor abnormalities in patients with OCD were task dependent. We found significant group $\times$ task interactions for the mean number of changes of direction of velocity per stroke (circles $v$ letter triples $F=4.72 ; \mathrm{p}<0.05$ ) and mean peak velocity (circles $v$ sentence $F=9.61 ; \mathrm{p}<.01$; circles $v$ signature $F=9.72$; 
A

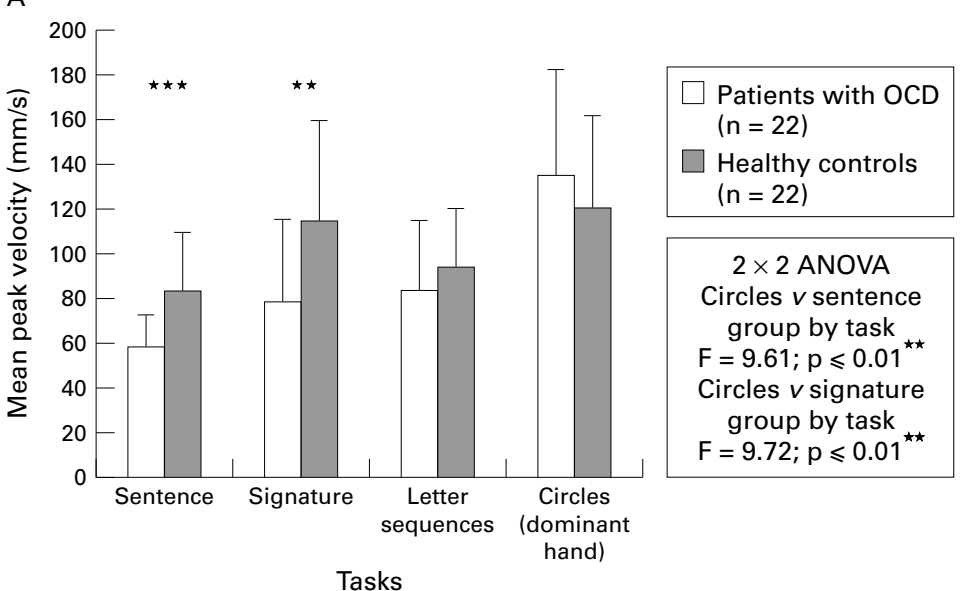

B

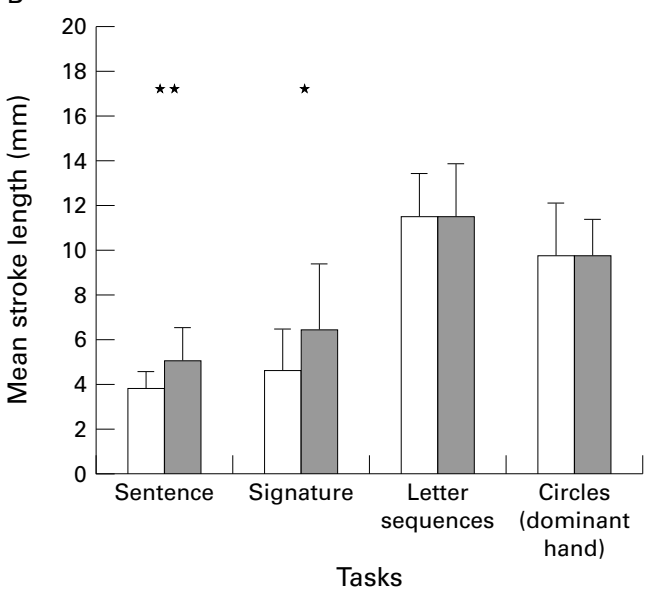

C

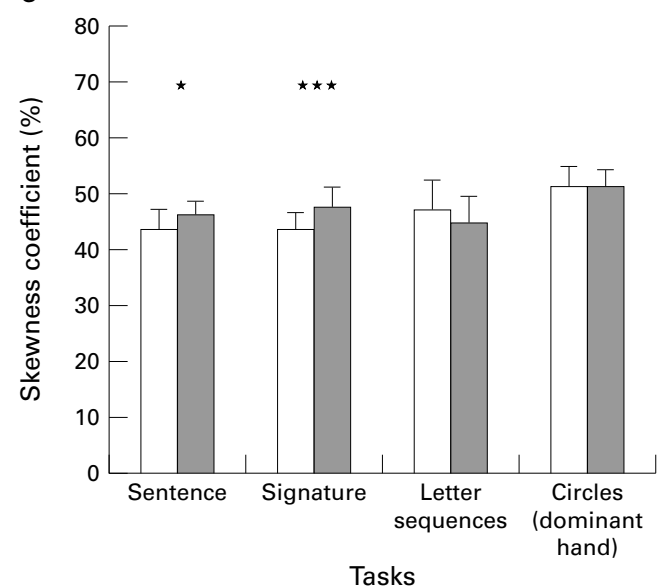

Figure 2 Differences between patients with OCD and healthy controls in performing different drawing and handwriting tasks regarding the degree of hand movement automatisation assessed with $(A)$ mean peak velocity, $(B)$ mean stroke length, and $(C)$ the skewness coefficient reflecting time to peak velocity per stroke.

$\mathrm{p}<0.01$ ), indicating that patients with OCD manifested faster and better automatised hand movements than healthy controls while performing simple motor tasks (repetitive drawing of superimposed concentric circles), but showed significantly slower handwriting movements (sentence and signature) and a lower degree of automatisation when writing letter sequences. Micrographia in patients with OCD was limited to handwriting (writing a sentence and personal signature); this fact was reflected by two group $\times$ task interactions, both significant at the $10 \%$ level (circles $v$ sentence $F=3.4$, $\mathrm{p}<0.10$; circles $v$ signature $F=4.31, \mathrm{p}<0.05$ ).

DIFFERENCES BETWEEN PATIENTS WITH OCD AND HEALTHY CONTROLS IN HAND MOTOR PERFORMANCE LATERALISATION

Overall, the multivariate variance analyses, using the kinematic parameters for the right and the left hand as the dependent variables and the group (patients with OCD $v$ controls) as the between subject factor, disclosed neither significant main effects for groups nor any significant group $\times$ side effects reflecting anomalous functional asymmetries.

CORRELATIONS WITH CLINICAL FACTORS

The $\mathrm{p}$ values for the correlational data have been adjusted considering each of the major clinical variables, for each task separately. The adjusted significance level was $\mathrm{p}=0.025(0.10 / 4$ (number of the assessed parameters)) for the tasks reflecting the degree of fine motor diadochokinesia and $p=0.017(0.10 / 6)$ for the handwriting tasks.

\section{AGE AT THE ONSET OF DISEASE}

The effect of age at the beginning of disease on kinematic parameters was assessed with Pearson's correlations and partial correlations controlling for age at the time of investigation. The higher the age at the onset of OCD was, the higher the number of changes in direction of velocity per stroke for the repetitive writing of letter sequences (Pearson's $r=0.69 ; \mathrm{p}=0.000$ ), the lower the mean peak velocity for the performance of the same task (Pearson's $r=-0.48 ; \mathrm{p}=0.03$ ), and the lower the peak velocity inconsistency for sentence and signature (Pearson's $r=-0.46 ; \mathrm{p}=0.03$ ). If the kinematic effects of age and severity of OCD (Y-BOCS total score) were controlled for, we found that the higher the age at the beginning of the disease, the higher the number of changes in the direction of velocity for letter sequences $(r=0.60 ; p=0.005)$ and the lower the peak velocity inconsistency for the writing of a given sentence $(r=-0.55 ; \mathrm{p}=0.011)$. By contrast, no such significant changes in the signing of the signature $(r=0.09 ; \mathrm{p}=0.719)$ remained after controlling for age. In addition, higher age onset correlated with lower stroke length inconsistency for the written sentence (partial $r=-0.55 ; \mathrm{p}=0.012$ ).

\section{SEVERITY OF DEPRESSION}

The higher the severity of depression measured by the total HDRS, the lower the degree of automatisation (number of changes of direction of velocity per stroke) for the repetitive drawing of circles with the dominant hand (Spearman's $r=0.52 ; p=0.014$ ). There was no significant correlation with any of the other kinematic parameters. Therefore we only controlled for the effects of depression when investigating the relation between the severity of OCD symptoms and the above mentioned parameter. 
SEVERITY OF OCD SYMPTOMS

Scores on the Y-BOCS were correlated with kinematic parameters by Spearman's analysis.

The higher the total Y-BOCS score, the higher the mean number of changes in direction of velocity per sentence stroke (Spearman's $r=0.52 ; \mathrm{p}=0.013$ ) and the higher some parameters reflecting the kinematics of signature (mean peak velocity and mean stroke length: Spearman's $r=0.46 ; \mathrm{p}=0.03$; skewness coefficient: Spearman's $r=0.56 ; \mathrm{p}=0.007$ ). Compulsions correlated in a more positive significant manner with the number of changes of direction of sentence stroke than did the total OCD score itself (Spearman's $r=0.69$; $\mathrm{p}=0.000)$. On the other hand, the higher the degree of obsessions was, the longer the time spent in the acceleration phase for the single writing strokes in the letter sequence task (Spearman's $r=0.48 ; \mathrm{p}=0.03$ ) was and the higher the peak velocity inconsistency regarding repetitive drawing of concentric circles with the non-dominant hand was (Spearman's $r=0.55 ; \mathrm{p}=0.008)$.

\section{Discussion}

Based on findings of motor disturbances in patients with $\mathrm{OCD}^{7}$ we had hypothesised that patients with OCD would write and draw slower and with a lower degree of automatisation than healthy controls. For writing, we could demonstrate significantly lower peak velocity, paired with shortened periods of acceleration per stroke, and micrographia in patients with OCD compared with controls, but not lower handwriting automatisation. We found no significant differences between groups in any other motor tasks. Therefore, our first hypothesis, implying task independent hand movement disorders in patients with OCD, must be rejected.

Our results suggest that poorer hand motor performance is restricted to handwriting tasks in OCD. In very simple motor tasks, patients with OCD achieved even higher peak velocity than controls. This finding is in line with the assumption that, due to basal ganglia dysfunction, strongly overlearned primitive behaviour patterns can be elicited faster in patients with OCD than in controls. ${ }^{31}$

It is of interest to compare the kinematic profile of handwriting movements in patients with OCD with that of patients with basal ganglia disorders (Parkinson's disease (PD) and Huntington's disease (HD)), as several findings $^{56}$ lend support to the assumption that basal ganglia dysfunction is a relevant factor in the aetiology of OCD..$^{32}$ Patients with PD have been found to write significantly more slowly and smaller than controls ${ }^{33}$; their hand movement consistency has been reported to be nor$\mathrm{mal}^{34}$; their acceleration phases were significantly longer than in age matched healthy subjects. ${ }^{35}$ Patients with HD showed significantly lower and less consistent velocity and a tendency for less consistent stroke length while writing. ${ }^{36}$ Stroke length and the skewness coefficient have been reported to be normal in $\mathrm{HD}^{37}$
The kinematic profile of handwriting movements in patients with OCD has more in common with that of patients with PD than those with HD. However, different handwriting tasks were used in the studies mentioned above: The drawing of cursive $1 s^{183036}$ versus the writing of a sentence and a signature in this study, requiring more motor control. Therefore, it may be argued that diseases of the basal ganglia are associated with severe motor impairment in even simple tasks, whereas hand motor disturbances of patients with OCD are discrete and can only be detected by computerised methods.

Comparing motor asymmetries in patients with OCD and controls, no significant differences were found. However, some studies suggest the lateralisation of neurological soft signs to the right cerebral hemisphere in OCD. ${ }^{12}{ }^{38} \mathrm{It}$ must be emphasised that our results are restricted to hand motor function; they do not exclude the possibility that cerebral dysfunction in patients with OCD is lateralised, as the areas of brain dysfunction may differ from those brain structures which play an important part in the control of hand movements.

There are some hints that an early age of OCD onset is linked to a pathological mechanism different from that of a later OCD onset (adulthood): Patients with an early onset are more likely to have Tourette's syndrome in their families, especially if OCD is associated with tics. $^{39}$ In this case, soft motor signs, reflecting a disturbance of very simple motor programmes, ${ }^{40}$ would be expected to be more pronounced in patients with early onset. We found less automatisation for letter sequences with later OCD onset, an indicator of "clumsy" velocity profiles per stroke for unusual tasks. By contrast, patients with an earlier OCD onset disclosed less consistent peak velocity and stroke length profiles for the writing of a sentence. Therefore, it can be concluded that an earlier OCD onset goes along with less regular handwriting, reflecting a disturbance of movement stroke sequencing, whereas later OCD onset is more often associated with discrete deviant stroke features, apparent during the performance of hand movements requiring more complex motor control.

The effects of the severity of depression on the kinematics of handwriting in patients with OCD were relatively low, although some patients had high HDRS scores and some kinematic studies ${ }^{17}{ }^{41}$ have documented fine motor retardation in depression. However it must be noted that Hollander et $a l^{12}$ did not find any significant correlation between the severity of depression and neurological soft signs in patients with OCD.

The correlation of the severity of OCD symptoms with disturbances in hand motor performance in OCD is of special interest, as Hollander $e t ~ a l^{12}$ have found increased rates of neurological soft signs in patients with OCD with a greater severity of obsessions. In our sample, the severity of compulsions was found to be associated with lower handwriting automatisation. Kinematic effects of obsessions were only found for unusual tasks: Significantly 
lower peak velocity consistency for rapid alternating movements of the non-dominant hand and a tendency for prolonged acceleration phases (writing of letter sequences) with more severe obsessions.

Our study has shown that kinematic investigations of soft motor signs can contribute important information about the possible role of neurological soft signs in OCD. It could be argued that patients with OCD adopt a qualitatively and kinematically different style of handwriting, because they are simply unable to confirm that they are performing the task adequately. This might more accurately reflect their psychopathology (for example, doubt, checking) than any fundamental difficulty in hand motor control. This hypothesis is plausible. However, we did not find significantly lower handwriting automatisation in patients with OCD, as would be expected if they checked their handwriting performance.

There are some restrictions limiting our conclusions:

(1) As our sample size is small, we cannot exclude that, due to type II errors, we may have failed to detect additional soft motor signs in patients with OCD. Similarly, sample size prevented a comparison of the degree of hand motor dysfunction in different OCD subgroups. However, we have found plausible kinematic findings in OCD that are suited for the generation of hypotheses that should be tested in larger samples.

(2) We have not directly compared patients with OCD with other psychiatric or neurological patient groups. In the future, studies will focus on the question of whether the kinematic analysis of soft motor signs can help distinguish OCD from other anxiety disorders, such as panic disorder or phobia.

We acknowledge Nico Niedermeier and Walter Hauke for their assistance in the psychopathological characterisation of the patients with OCD recruited for this study. We express our gratitude to Julian Rihl and Marlies Karsch for their examination of hand motor performance in the healthy controls. Particularly the patients with OCD and the healthy subjects are gratefully acknowledged for their readiness to participate in ou study.

\section{Appendix: Vignette of a patient with OCD showing a low degree of handwriting automatisation (fig 1 )}

This 56 year old man gave a 3 year history of superstitious behaviour patterns, aggressive obsessions (fears of injuring other people), excessive dealing with moral problems and the correctness of personal actions, magical thoughts (superstitious fears, numbers associated with fortune or the opposite, colours with special meanings), and perpetual checking for his mistakes. The first treatment of symptoms of OCD had been 3 years earlier. At the time of assessment he was not taking any medication. At the time of testing, his above mentioned obsessions were severe (Y-BOCS score ${ }^{22-23}$ for obsessions 19) and his compulsions were of moderate intensity (Y-BOCS score ${ }^{22-23}$ for compulsions 12 ). The physician examining him assessed him to be very severely ill, his degree of concomitant depression as measured using the HDRS total score $(7)^{24}$ being quite low. The patient's verbal intelligence as measured by his WST IQ $(125)^{20}$ was high, reflecting his high educational level (successful studies as a science student at a university). It is of interest that the patient was unable to perform this test in the original paper and pencil form because of his obsessions to control his activities; instead the instructor had to read the word lists with a loud voice and the patient told him which words he thought did or did not make sense. His physical examination showed no signs of headache, restlessness, agitation, hypokinesia, rigour, tremor, or myoclonies.

1 Rasmussen SA, Eisen JL. Epidemiology of obsessivecompulsive disorder. $\mathcal{F}$ Clin Psychiatry 1990;51(suppl.):1013.

2 Hegerl U, Mavrogiorgou P. The biological basis of OCD. Verhaltensther Verhaltensmed 1999;20:435-47.

3 Luxenberg JS, Swedo SE, Flament MF, et al. Neuroanatomical abnormalities in obsessive-compulsive disorder detected with quantitative $\mathrm{x}$-ray computed tomography. Am f Psychiatry 1988;145:1089-93.

4 Robinson D, Wu H, Munne RA, et al. Reduced caudate nucleus volume in obsessive-compulsive disorder. Arch Gen Psychiatry 1995;52:393-8.

5 Rapoport JL, Swedo SE, Leonard HL. Childhood obsessive compulsive disorder. F Clin Psychiatry 1992;53(suppl):1116.

6 Pauls DL, Towbin KE, Leckman JF, et al. Gilles de la Tourette's syndrome and obsessive-compulsive disorder. Tourette's syndrome and obsessive-compulsive disorder.
Evidence supporting a genetic relationship. Arch Gen Evidence supporting a gene

7 Yaryura-Tobias JA, Stevens K, Neziroglu F. Motor disturbances in the obsessive compulsive disorder and its pectrum. Neurol Psychiatry Brain Res 1997;5:79-84.

8 Hermesh H, Hoffnung RA, Aizenberg D, et al. Catatonic signs in severe obsessive compulsive disorder. I Clin Psychiatry 1989;50:303-5.

9 Rachman S. Primary obsessional slowness. Behav Res Ther 1974;12:9-18.

10 Hymas NFS, Lees A, Bolton D, et al. The neurology of obsessional slowness. Brain 1991;114:2203-33.

11 Yaryura-Tobias JA, Neziroglu FA. Obsessive-compulsive disorders of childhood. In: Yaryura-Tobias JA, Neziroglu FA, eds. The obsessive-compulsive disorder spectrum. Washington, DC: American Psychiatric Association 1997:79-92.

12 Hollander E, Schiffman E, Cohen B, et al. Signs of central nervous system dysfunction in obsessive-compulsive disorder. Arch Gen Psychiatry 1990;47:27-32.

13 Hollander E, DeCaria CM, Saoud JB, et al. Neurological soft signs in obsessive-compulsive disorder. Arch Gen soft signs in obsessive-com
Psychiatry 1991;48:278-9.

14 Nickoloff SE, Radant AD, Reichler R, et al. Smooth pursuit and saccadic eye movements and neurological soft signs in obsessive-compulsive disorder. Psychiatry Res 1991;38: $173-85$

15 Mergl R, Tigges P, Schröter A, et al. Digitized analysis of handwriting and drawing movements in healthy subjects. Methods, results and perspectives. If Neurosci Methods 1999;90:157-69.

16 Tigges P, Mergl R, Frodl T, et al. Digitized analysis of abnormal hand-motor performance in schizophrenic patients. Schizophr Res 2000;45:133-43.

17 Sabbe B, Hulstijn W, van Hoof JJM, et al. Fine motor retardation and depression. F Psychiatr Res 1996;30:295-306.

18 Slavin MJ, Phillips JG, Bradshaw JL, et al. Consistency of handwriting movements in dementia of the Alzheimer's type: a comparison with Huntington's and Parkinson's distype: a comparison with Huntington's and

19 Oldfield RC. The assessment and analysis of handedness: the Edinburgh inventory. Neuropsychologia 1971;9:97-113. Schmidt KH, Metzler P. Wortschatztest. Weinheim: Beltz, 1992 .

21 World Medical Association, eds. World Medical Association declaration of Helsinki. Recommendations guiding physicians in biomedical research involving human subjects. fAMA 1997;277:925-6.

22 Goodman WK, Price LH, Rasmussen SA, et al. The Yale-Brown obsessive compulsive scale. I. Development, use and reliability. Arch Gen Psychiatry 1989;46:1006-11.

23 Goodman WK, Price LH, Rasmussen SA, et al. The Yale-Brown obsessive compulsive scale. II. Validity. Arch Gen Psychiatry 1989;46:1012-16.

24 Guy W. ECDEU assessment manual for psychopharmacology. Washington, DC: Department of Health, Education and Welfare, 1976

25 Beck AT, Ward CH, Mendelson M, et al. An inventory for measuring depression. Arch Gen Psychiatry 1961;4:561-71.

26 Marquardt C, Mai N. CS-Computerunterstützte Analyse der Marquardt C, Mai N. CS - Computerunterstützte Analyse der
Bewegungsabläufe beim Schreiben. Bedienungshandbuch. Bewegungsablaufe beim Schre

27 Jahn T. Diskrete motorische Störungen bei Schizophrenie. Weinheim: Beltz, 1999

28 Marquardt C, Mai N. A computational procedure for movement analysis in handwriting. $\mathcal{F}$ Neurosci Methods 1994;52: $39-45$.

29 Eichhorn T, Gasser T, Mai N, et al. Computational analysis of open loop handwriting movements in Parkinson's disease: a rapid method to detect dopamimetic effects. Mov Disord 1996;11:289-97.

30 Gallucci RM, Phillips JG, Bradshaw JL, et al. Kinematic analysis of handwriting movements in schizophrenic patients. Biol Psychiatry 1997;41:830-3.

31 Lesch KP. Psychobiologie der Zwangsstörung. Fortsch Neurol Psychiatr 1991;59:404-12.

32 Rapoport JL. Obsessive compulsive disorder and basal ganglia dysfunction. Psychol Med 1990;20:465-9.

33 Margolin DI, Wing AM. Agraphia and micrographia: clinical manifestations of motor programming and performance disorders. Acta Psychol (Amst) 1983;54:263-83. 
34 Phillips JG, Stelmach G, Teasdale N. What can indices of handwriting quality tell us about parkinsonian handwrithandwriting quality tell us about part

35 Phillips JG, Stelmach G, Teasdale N. Preliminary assessment of spatiotemporal control of handwriting in parkinsoment of spatiotemporal control of handwriting in parkinso-
nians. In: Plamondon R, Suen CY, Simner ML, eds. Computer recognition and human production of handwriting. Singapore: World Scientific 1989:317-31.

36 Phillips JG, Bradshaw JL, Chiu E, et al. Characteristics of handwriting of patients with Huntington's disease. Mov Disord 1994:9:521-39.

37 Phillips JG, Chiu E, Bradshaw JL, et al. Impaired movement sequencing in patients with Huntington's disease: a kinematic analysis. Neuropsychologia 1995;33:365-9.
38 Behar D, Rapoport JL, Berg C, et al. Computerized tomography and neuropsychological test measures in adolescents with obsessive-compulsive disorder. Am F Psychiatry 1984; 141:363-9.

39 Pauls DL, Alsobrook II JP, Goodman W, et al. A family study of obsessive-compulsive disorders. Am F Psychiatry 1995;152:76-84.

40 Cummings JL, Frankel M. Gilles de la Tourette syndrome and the neurological basis of obsessions and compulsions. Biol Psychiatry 1985;20:117-26.

41 Sabbe B, Hulstijn W, van Hoof J, et al. Retardation in depression: assessment by means of simple motor tasks. $\mathcal{F}$ Affect Disord 1999;55:39-44.

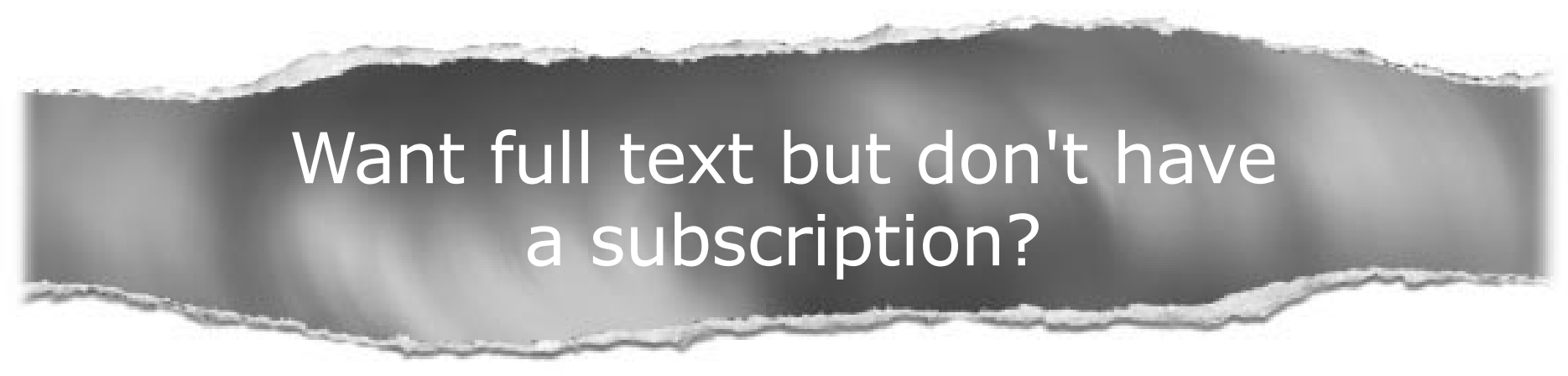

\section{Pay per view}

For just \$8 you can purchase the full text of individual articles using our secure online ordering service. You will have access to the full text of the relevant article for 48 hours during which time you may download and print the pdf file for personal use.

\section{www.jnnp.com}

